\title{
A CASE STUDY OF DARK GRB 051008
}

\author{
A. Volnova ${ }^{1,2}$, A. Pozanenko ${ }^{2}$, J. Gorosabel ${ }^{3,4,5}$, D. Perley ${ }^{6}$, D.A. Kann ${ }^{7}$, \\ D. Frederiks ${ }^{8}$, V. Rumyantsev ${ }^{9}$, A.J. Castro-Tirado ${ }^{3}$ and P. Minaev ${ }^{2}$
}

\begin{abstract}
We present multi-wavelength observations of the dark GRB 051008. The burst was not detected in the optical bands, however we discover the host galaxy and secured the redshift of the host with following campaign of multicolor observations of Shajn, NOT, Gemini North and Keck telescopes. We provide arguments that the galaxy could be in a complex of gravitationally bound galaxies. Our investigation of the GRB 051008 also confirms a tendency of host galaxies of dark bursts to be more dusty.
\end{abstract}

\section{Observations and results}

GRB 051008 was detected by the Swift/BAT at 16:33:21 UT on October 8, 2005 (Marshall et al. 2005). It was also detected in $\gamma$-ray domain by Suzaku/WAM (Ohno et al. 2005), Konus-WIND, and INTRGRAL/SPI-ACS. The Swift/XRT discovered an X-ray counterpart in $\sim 50$ minutes after the trigger because of the Earth constraint. 2.6-meter Shain telescope of Crimean observatory (ZTSh/CrAO)

1 Sternberg Astronomical Institute of Lomonosov Moscow State University, Moscow, Russia

2 Space Research Institute of the Russian Academy of Sciences, Moscow, Russia

3 Instituto de Astrofísica de Andalucía (IAA-CSIC), Glorieta de la Astronomía s/n, 18008, Granada, Spain

4 Unidad Asociada Grupo Ciencia Planetarias UPV/EHU-IAA/CSIC, Departamento de Física Aplicada I, E.T.S. Ingeniería, Universidad del PaísVasco UPV/EHU, Alameda de Urquijo s/n, 48013 Bilbao, Spain

5 Ikerbasque, Basque Foundation for Science, Alameda de Urquijo 36-5, 48008 Bilbao, Spain

${ }^{6}$ Department of Astronomy, California Institute of Technology, Pasadena, USA

7 Thüringer Landessternwarte Tautenburg, Tautenburg, Germany

8 Ioffe Physico-Technical Institute of the Russian Academy of Sciences, St. Petersburg, Russia

9 Scientific Research Institute Crimean Astrophyscial Observatory, Nauchny, Ukraine

*e-mail: alinusss@gmail.com 
did not detect any optical afterglow up to $23.3^{\mathrm{m}}$ in $\sim 30$ minutes after the trigger (Rumyantsev et al. 2005). Together with XRT observations this allows to conclude that GRB 051008 is an optically dark burst with $\beta_{O X}<0.18$. In Apr. 2006 the host galaxy of the source with R-band magnitude of $23.9^{\mathrm{m}}$ was discovered by ZTSh/CrAO (Fig. 1, left). The probability for an accidental location of the Id3 galaxy inside the XRT error circle is estimated to be of about $1.96 \%$. In 2005 - 2012 the observations of the host galaxy were carried out with Shain, Tautenburg, NOT, Gemini North, and Keck I telescopes in $U, B, g^{\prime}, V, R, i$, $I, Z$ and $K^{\prime}$ bands down to limiting magniudes 25.4, 25.8, 27.2, 25.7, 26.1, 23.9, $25.2,25.5$, and 23.0 correspondingly. A long slit spectrum of the host galaxy was obtained in Jun. 2009 using the Low-Resolution Imaging Spectrometer (LRIS) on the Keck I telescope, no obvious line features are identified in the sky-subtracted $2 \mathrm{D}$ spectra.

The multiwavelength photometry was used to estimate the photometric redshift $z_{\text {phot }}$ of the host galaxy and 4 nearby galaxies with LePhare package (Arnouts et al. 1999; Ilbert et al. 2006). We used the COSMOS population synthesis models library to obtain the best-fitted SED with emission lines and the redshift (Fig. 1, right). We used the PEGASE2 population synthesis models library with to obtain all the other required parameters. The host galaxy is best described by a template of a starburst galaxy with SMC reddening law at the redshift $z_{\text {phot }}=2.85_{-0.05}^{+0.03}$ with the age of the dominant stellar population being about $0.025 \mathrm{Gyr}$ and an intrinsic extinction of $A_{V}=0.23^{\mathrm{m}}$ and SFR about $80-100 M_{\odot} / \mathrm{y}$. The redshift of the galaxy Id5 (see Fig.1, left) is $z=2.84_{-0.03}^{+0.02}$, it corroborates the hypothesis that the host galaxy is located in a gravitationally bound at least pair of galaxies with a distance of about $70 \mathrm{kpc}$.

GRB 051008 is a good example of a multi-peak event. Using SPI-ACS data we found at least 10 separated pulses which fit the light curve fairly well. Short duration peaks of the burst may be the cause of negligible spectral lag of $-0.2 \pm 0.3$ defined from Konus-WIND data (between G1 and G3 channels).

We fitted the $\mathrm{X}$-ray spectrum $\sim 4$ hours after the trigger with single power-law model absorbed by the Galactic $N_{H}$ and $N_{H, h o s t, z}$ of the host galaxy with fixed redshift. This model yields an X-ray photon index $\Gamma_{X} \sim 1.9$ and $N_{H, \text { host, } z}=$ $12.2 \times 10^{21} \mathrm{~cm}^{-2}$ corresponding to the extinction along the line of sight $A_{V, G R B}=$ $5.5^{\mathrm{m}} \pm 0.2^{\mathrm{m}}$ (Güver \& Özel 2009). This line-of-sight extinction can not be explained neither by the bulk absorption in the host galaxy nor by adding of a cooling break $\Delta \beta=0.5$ in the energy range lower than the XRT band.

Swift/BAT, INTEGRAL/SPI-ACS, and Swift/XRT data were converted into $15-50 \mathrm{keV}$ range in order to construct a joint X-ray light curve which was fitted by a broken power-law (Liang et al. 2007). The light curve of GRB 051008 X-ray afterglow belongs to a less frequent class of plateau-less XRT light curves and may represent only phases III and IV of the canonical X-ray afterglow (Racusin et al. 2009). The time of the light curve break $t_{b}$ and $z_{\text {phot }}$ allowed to estimate the isotropic equivalent energy for the burst $E_{\text {iso }}=(11.17 \pm 3.69) \times 10^{53} \mathrm{erg}$, the jet opening angle, and the total gamma radiation energy in case of constant ISM with the density of $n=1 \mathrm{~cm}^{-3}$ (Zhang et al. 2007) $\left(\theta_{j}=1.7^{\circ} \pm 0.2^{\circ}\right.$, 

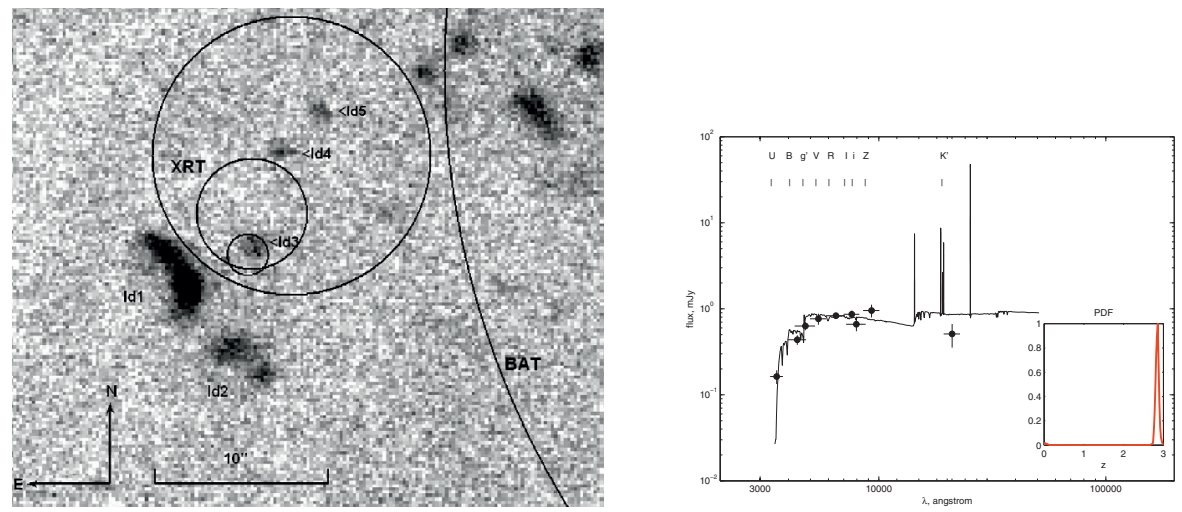

Fig. 1. Left: GRB 051008 and its immediate neighbourhood. The image was taken 255 days after the GRB in the R-filter, a 4800 s exposure, the NOT telescope. The burst error circles determined from the Swift/BAT and the Swift/XRT telescopes data are shown with solid lines. The smallest error box depicts the Swift/XRT refined error circle (Goad et al. 2007). The Id3 marker indicates the host galaxy of GRB 051008. Right: spectral Energy Distribution (SED) of the host galaxy in the observer frame. The bestfitted SED type obtained by LePhare (line) is the SED of starburst galaxy on $z=2.85$. Observed flux in $U B g^{\prime} V R i I Z K^{\prime}$ filters is shown by crosses. The associated Probability Distribution Functions is shown on the enclosed panel. The SED with emission lines is obtained using COSMOS SED library and SMC reddening law (Prévot et al. 1984).

$\left.E_{\gamma}=(4.6 \pm 2.0) \times 10^{50} \mathrm{erg}\right)$ and wind-like environment (Chevalier \& Li 2000) $\left(\theta_{j}=2.0^{\circ} \pm 0.2^{\circ}, E_{\gamma}=(6.8 \pm 4.4) \times 10^{50} \mathrm{erg}\right)$.

GRB 051008 is dark neither due to high redshift nor due to bulk extinction in the host galaxy, which is moderate only. We suggest that the darkness of the burst is most probably due to a local extinction of the molecular cloud surrounding the progenitor of the burst. While the burst well fits Amati relation (Amati 2006), it is an outlier of Ghirlanda relation (Ghirlanda et al. 2004). Assuming that the break on the X-ray light curve is a real jet-break the latter can indicate a dense $\left(n=10^{3}-10^{6} \mathrm{~cm}^{-3}\right)$ environment of the burst but it can also.

The work was partially supported by RFBR grants 12-02-01336-a, 11-01-92202-Mong-a.

\section{References}

Amati, L., 2006, MNRAS, 372, 233

Arnouts, S., Cristiani, S., Moscardini, L., et al., 1999, MNRAS, 310, 540

Chevalier, R.A., \& Li, Z.-Y., 2000, ApJ 536, 195

Ghirlanda, G., Ghisellini G., \& Lazzati D., 2004, ApJ, 616, 331 
Goad, M.R., Tyler, L.G., Beardmore, A.P., et al., 2007, A\&A, 476, 1401 Güver, T., \& Özel, F., 2009, MNRAS, 400, 2050

Ilbert, O., Arnouts, S., McCracken, H.J., et al., 2006, A\&A, 457, 841

Liang, E.-W., Zhang, B.-B., \& Zhang, B., 2007, ApJ, 670, 565

Marshall, F., Barthelmy, S., Cummings, J., et al., 2005, GCN Circ. 4069

Ohno, M., Takahashi, T., Fukazawa, Y., et al., 2005, GCN Circ. 4297

Prévot, M.L., Lequeux, J., Prévot, L., et al., 1984, A\&A, 132, 389

Racuzin, J., Liang, E.W., Burrows, D.N., et al., 2009, ApJ, 698, 43

Rumyantsev, V., Biryukov, V., \& Pozanenko, A., 2005, GCN Circ. 4081

Zhang, B., Liang, E., Page, K.L., et al., 2007, ApJ, 655, 989 